Article

\title{
Genome-Wide Identification and Analysis of U-Box E3 Ubiquitin-Protein Ligase Gene Family in Banana
}

\author{
Huigang Hu, Chen Dong, Dequan Sun, Yulin Hu and Jianghui Xie * \\ Key Laboratory of Tropical Fruit Biology, Ministry of Agriculture, South Subtropical Crop Research Institute, \\ Chinese Academy of Tropical Agricultural Science, Zhanjiang 524091, China; \\ zjhuhuigang@163.com/huhuigang@sina.com (H.H.); nysdongchen1981@163.com (C.D.); \\ sscrisun@163.com (D.S.); huyulin2003@126.com (Y.H.) \\ * Correspondence: xiejianghui168@163.com; Tel.: +86-759-2859272
}

Received: 18 October 2018; Accepted: 1 December 2018; Published: 4 December 2018

check for updates

\begin{abstract}
The U-box gene family is a family of genes which encode U-box domain-containing proteins. However, little is known about U-box genes in banana (Musa acuminata). In this study, 91 U-box genes were identified in banana based on its genome sequence. The banana U-box genes were distributed across all 12 chromosomes at different densities. Phylogenetic analysis of U-box genes from banana, Arabidopsis, and rice suggested that they can be clustered into seven subgroups (I-VII), and most U-box genes had a closer relationship between banana and rice relative to Arabidopsis. Typical U-box domains were found in all identified MaU-box genes through the analysis of conserved motifs. Four conserved domains were found in major banana U-box proteins. The MaU-box gene family had the highest expression in the roots at the initial fruit developmental stage. The MaU-box genes exhibited stronger response to drought than to salt and low temperatures. To the best of our knowledge, this report is the first to perform genome-wide identification and analysis of the U-box gene family in banana, and the results should provide valuable information for better understanding of the function of U-box in banana.
\end{abstract}

Keywords: banana; ubiquitin-protein ligases; gene family; fruit development; abiotic stresses

\section{Introduction}

The ubiquitin/26S proteasome (UPS) pathway degrades ubiquitinated substrate proteins and is extensively involved in various cellular processes [1]. The diverse aspects of plant growth and development and the degradation of short-lived regulatory proteins can be regulated by the UPS [2-4]. E1 Ub-activating enzyme, E2 Ub-conjugating enzyme, and E3 Ub ligase are necessary for ubiquitin activation and transfer [5]. First, E1 activates the ubiquitin molecule in an ATP-dependent manner, and then E2 facilitates the attachment of ubiquitin molecule to the target protein in the presence of E3 [6]. E3 ligase plays an important role in protein ubiquitination because E3 can identify target proteins for modification [7]. A single protein or a protein complex joins the ubiquitin reaction, which could be conferred by E3 ligase [8,9]. Ubiquitin E3 ligases facilitate the covalent attachment of ubiquitin to target proteins in eukaryotes [10]. HECT, RING finger, and U-box domain proteins are three types of single-protein E3 ligases [11]. U-box proteins are found in yeast, plants, and animals [12-15]. The U-box domain is composed of approximately 75 amino acids (aa) [16,17]. Many U-box proteins had function of E3 ligases [18,19]. The genome of Arabidopsis thaliana has more than 60 U-box genes, which have many functions in plants [16]. A previous study has identified the functions of U-box E3 ligases in parsley, tomato, tobacco, and rice [20]. OsU-box gene 51 negatively regulates cell death signaling according to cell death assay [17]. The U-box E3 ligase NtACRE276 of tobacco may play a role in Cf9/Avr9-elicited defense [21]. On the basis of protein domains, eight groups of Plant U-box 
(PUB) genes are present in the 125 PUB genes of soybean [22]. The flowering condition could be changed in GmPUB8-overexpressing Arabidopsis, which flowered earlier under middle- and short-day conditions but later under long-day conditions [22]. Inactivation of the Arabidopsis PUB13 also results in spontaneous cell death, enhanced levels of the defence hormone SA, and early flowering [23]. In grapevine, the PUB gene significantly regulates the accumulation of resistance proteins under both biotic and abiotic stresses [20].

Banana (Musa spp.) is one of the world's most important fruits [24,25]. The sequencing of the whole genome of banana (Musa acuminata) provides a good platform for the development of banana molecular biology [26]. Until now, the U-box gene family of banana is rarely studied. U-box genes may play important roles in the growth and development of banana, so investigating the E3 gene family in banana is necessary. In this study, the whole genome of the banana U-box gene was determined and analyzed. The conserved domain structure, subgroup classification, evolutionary relationship, intron and exon structure, gene expansion, chromosome mapping, and expression profile analysis were studied, providing a theoretical basis for the analysis of U-box gene functions.

\section{Results}

\subsection{Identification and Chromosomal Localization of $U$-Box Gene Family Members}

In this study, 91 PUB genes are found in banana genome (Table 1). The MaU-box protein contains a 60-70 aa U-box conserved domain. The length of MaU-box was from 660 (MaU-box69) to $6279 \mathrm{bp}$ (MaU-box57), and the average length was $1789 \mathrm{bp}$. The predicted protein product range was 219-2092 aa, with an average length of 595 aa. The relative molecular weight (MW) ranged from $23.38 \mathrm{kD}$ to $223.93 \mathrm{kD}$, with an average of 64.85. The isoelectric point (PI) was in the range of 4.96 (MaU-box78) to 9.57 (MaU-box13). Subcellular localization analysis indicated that $93 \%$ of the MaU-box proteins were located in the nucleus and that only six were located in the cytoplasm (Table 1). These findings suggested that the vast majority of MaU-box function in the nucleus.

A MaU-box chromosomal localization map was plotted (Figure 1). Ninety genes from 91 MaU-box genes were located on chromosomes. Chromosome 3, where the largest number of MaU-box genes was found, contained $11 \mathrm{MaU}$-box genes. It is followed by chromosomes 4, 5, and 11, which contained $10 \mathrm{MaU}-b o x$ genes. Nine MaU-box genes were located in chromosomes 7, 9, and 10; 7 MaU-box genes were found in chromosome 8; 6 MaU-box genes were observed in chromosome 1; 5 MaU-box genes were localized in chromosome 2; and only $4 \mathrm{MaU}$-box genes were detected in chromosome 11.

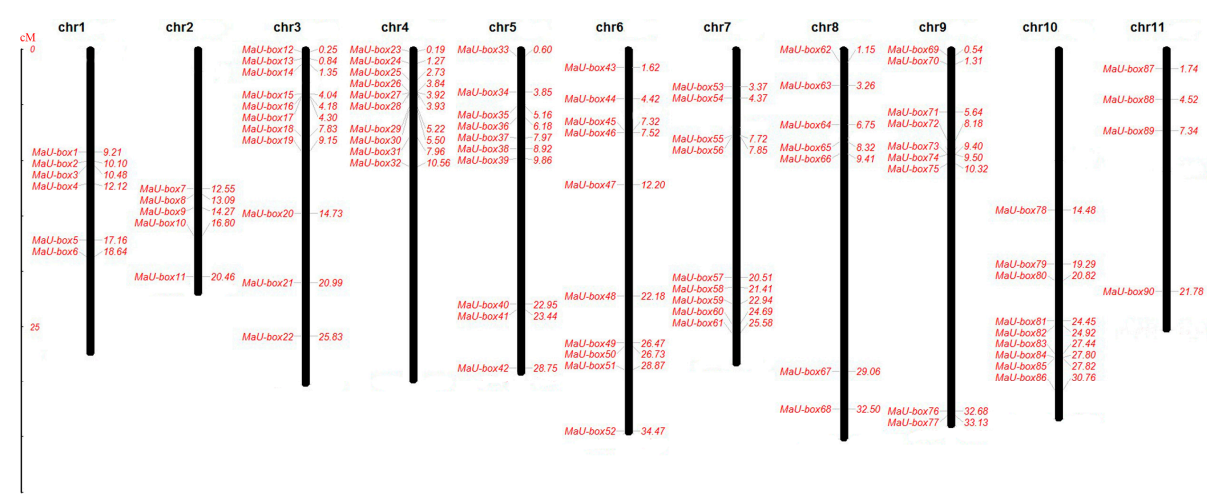

Figure 1. MaU-box chromosomal localization maps. The size of a chromosome is indicated by its relative length in centimorgan (cM). 
Table 1. The information of Plant U-box (PUB) gene family in banana

\begin{tabular}{|c|c|c|c|c|c|c|c|c|c|}
\hline $\begin{array}{l}\text { Gene } \\
\text { Name }\end{array}$ & Locus ID & Chromosome Location & $\begin{array}{c}\text { Gene } \\
\text { DNA (bp) }\end{array}$ & $\begin{array}{l}\text { CDS } \\
\text { (bp) }\end{array}$ & $\begin{array}{c}\text { Protein } \\
\text { Length (aa) }\end{array}$ & $\begin{array}{c}\text { Molecular } \\
\text { Weight (kD) }\end{array}$ & $\begin{array}{c}\text { Theoretical } \\
\text { pI }\end{array}$ & Exon & Putative Localization \\
\hline MaPUB1 & GSMUA_Achr1T12080_001 & chr1:9216255..9219037 reverse & 2783 & 1944 & 647 & 72.51 & 5.02 & 3 & Nucleus \\
\hline MaPUB2 & GSMUA_Achr1T13220_001 & chr1:10096902..10098167 forward & 1266 & 1191 & 396 & 41.13 & 7.66 & 2 & Nucleus \\
\hline MaPUB3 & GSMUA_Achr1T13730_001 & chr1:10477987..10483072 forward & 5086 & 1128 & 375 & 42.2 & 5.58 & 5 & Cytoplasm, Nucleus \\
\hline MaPUB4 & GSMUA_Achr1T16210_001 & chr1:12121706..12123412 reverse & 1707 & 1707 & 568 & 60.39 & 8.62 & 1 & Cytoplasm \\
\hline MaPUB5 & GSMUA_Achr1T22790_001 & chr1:17158846..17160507 reverse & 1662 & 1362 & 453 & 47.14 & 8.76 & 3 & Nucleus \\
\hline MaPUB6 & GSMUA_Achr1T24290_001 & chr1:18636205..18639761 reverse & 3557 & 1836 & 611 & 66.29 & 5.48 & 6 & Nucleus \\
\hline MaPUB7 & GSMUA_Achr2T08620_001 & chr2:12553612..12555419 reverse & 1808 & 1305 & 434 & 47.08 & 5.68 & 2 & Nucleus \\
\hline MaPUB8 & GSMUA_Achr2T09640_001 & chr2:13094735..13107492 forward & 12,758 & 2547 & 848 & 94.33 & 6.83 & 6 & Nucleus \\
\hline MaPUB9 & GSMUA_Achr2T11370_001 & chr2:14269037..14272568 forward & 3532 & 2301 & 766 & 84.56 & 5.85 & 4 & Nucleus \\
\hline MaPUB10 & GSMUA_Achr2T15530_001 & chr2:16801361..16803647 reverse & 2287 & 1983 & 660 & 72.39 & 9.22 & 3 & Nucleus \\
\hline MaPUB11 & GSMUA_Achr2T20690_001 & chr2:20462606..20463811 forward & 1206 & 1206 & 401 & 42.65 & 6.75 & 1 & Nucleus \\
\hline MaPUB12 & GSMUA_Achr3T00280_001 & chr3:253594..258827 forward & 5234 & 1812 & 603 & 65.43 & 5.52 & 5 & Nucleus \\
\hline MaPUB13 & GSMUA_Achr3T01110_001 & chr3:844229..845967 forward & 1739 & 1641 & 546 & 55.97 & 9.57 & 2 & Nucleus \\
\hline MaPUB14 & GSMUA_Achr3T02030_001 & chr3:1351349..1352952 reverse & 1604 & 1260 & 419 & 45.71 & 8.64 & 3 & Nucleus \\
\hline MaPUB15 & GSMUA_Achr3T06000_001 & chr3:4040242..4042320 reverse & 2079 & 1560 & 519 & 55.21 & 7.56 & 3 & Nucleus \\
\hline MaPUB16 & GSMUA_Achr3T06160_001 & chr3:4178177..4180306 reverse & 2130 & 1704 & 567 & 61.78 & 9.34 & 2 & Cytoplasm \\
\hline MaPUB17 & GSMUA_Achr3T06320_001 & chr3:4302726..4305879 reverse & 3154 & 2805 & 934 & 103.26 & 5.67 & 5 & Nucleus \\
\hline MaPUB18 & GSMUA_Achr3T10610_001 & chr3:7831098..7835605 reverse & 4508 & 2745 & 914 & 101.97 & 6.58 & 5 & Nucleus \\
\hline MaPUB19 & GSMUA_Achr3T12370_001 & chr3:9151629..9153329 reverse & 1701 & 1701 & 566 & 59.93 & 8.64 & 1 & Cytoplasm \\
\hline MaPUB20 & GSMUA_Achr3T14620_001 & chr3:14736420..14738258 reverse & 1839 & 1677 & 558 & 59.6 & 8.58 & 1 & Cytoplasm \\
\hline MaPUB21 & GSMUA_Achr3T19660_001 & chr3:20989179..20992456 forward & 3278 & 2946 & 981 & 107.65 & 5.92 & 4 & Nucleus \\
\hline MaPUB22 & GSMUA_Achr3T25750_001 & chr3:25831272..25832624 forward & 1353 & 1290 & 429 & 46.17 & 5.38 & 2 & Nucleus \\
\hline MaPUB23 & GSMUA_Achr4T00200_001 & chr4:193350..195464 reverse & 2115 & 2115 & 704 & 75.88 & 6.39 & 1 & Nucleus \\
\hline MaPUB24 & GSMUA_Achr4T01530_001 & chr4:1265636..1274901 reverse & 9266 & 1836 & 611 & 67.06 & 5.89 & 6 & Cytoplasm, Nucleus \\
\hline MaPUB25 & GSMUA_Achr4T03430_001 & chr4:2727458..2728362 reverse & 905 & 810 & 269 & 28.36 & 8.73 & 2 & Nucleus \\
\hline MaPUB26 & GSMUA_Achr4T04900_001 & chr4:3844084..3846449 forward & 2366 & 1587 & 528 & 59.15 & 5.65 & 7 & Nucleus \\
\hline MaPUB27 & GSMUA_Achr4T05020_001 & chr4:3920776..3926784 forward & 6009 & 2853 & 950 & 105.01 & 5.91 & 5 & Cytoplasm, Nucleus \\
\hline MaPUB28 & GSMUA_Achr4T05030_001 & chr4:3927558..3929980 reverse & 2423 & 1575 & 524 & 58.48 & 5.86 & 7 & Nucleus \\
\hline MaPUB29 & GSMUA_Achr4T06990_001 & chr4:5215355..5216775 forward & 1421 & 1212 & 403 & 41.42 & 8.56 & 3 & Nucleus \\
\hline MaPUB30 & GSMUA_Achr4T07400_001 & chr4:5497755..5499122 reverse & 1368 & 1305 & 434 & 47.46 & 5.63 & 2 & Nucleus \\
\hline MaPUB31 & GSMUA_Achr4T11070_001 & chr4:7963314..7965615 reverse & 2302 & 1269 & 422 & 43.29 & 5.56 & 2 & Nucleus \\
\hline MaPUB32 & GSMUA_Achr4T14130_001 & chr4:10562237..10563578 forward & 1342 & 1044 & 347 & 37.03 & 5.72 & 2 & Nucleus \\
\hline MaPUB33 & GSMUA_Achr5T01020_001 & chr5:597533..598908 forward & 1376 & 1206 & 401 & 42.81 & 8.42 & 2 & Nucleus \\
\hline MaPUB34 & GSMUA_Achr5T05220_001 & chr5:3854463..3856370 forward & 1908 & 1458 & 485 & 51.15 & 6.23 & 3 & Nucleus \\
\hline MaPUB35 & GSMUA_Achr5T07140_001 & chr5:5158762..5162462 forward & 3701 & 1887 & 628 & 69.15 & 5.75 & 7 & Nucleus \\
\hline MaPUB36 & GSMUA_Achr5T08500_001 & chr5:6179256..6185540 reverse & 6285 & 3129 & 1042 & 114.11 & 5.84 & 5 & Nucleus \\
\hline MaPUB37 & GSMUA_Achr5T11040_001 & chr5:7966157..7968849 reverse & 2693 & 2094 & 697 & 76.13 & 5.74 & 4 & Nucleus \\
\hline MaPUB38 & GSMUA_Achr5T12380_001 & chr5:8892024..8899046 forward & 7023 & 2403 & 800 & 90.49 & 6.39 & 12 & Nucleus \\
\hline
\end{tabular}


Table 1. Cont.

\begin{tabular}{|c|c|c|c|c|c|c|c|c|c|}
\hline $\begin{array}{l}\text { Gene } \\
\text { Name }\end{array}$ & Locus ID & Chromosome Location & $\begin{array}{c}\text { Gene } \\
\text { DNA (bp) }\end{array}$ & $\begin{array}{l}\text { CDS } \\
\text { (bp) }\end{array}$ & $\begin{array}{c}\text { Protein } \\
\text { Length (aa) }\end{array}$ & $\begin{array}{c}\text { Molecular } \\
\text { Weight (kD) }\end{array}$ & $\begin{array}{c}\text { Theoretical } \\
\text { pI }\end{array}$ & Exon & Putative Localization \\
\hline MaPUB39 & GSMUA_Achr5T13700_001 & chr5:9859570..9861021 forward & 1452 & 1365 & 454 & 48.23 & 8.75 & 1 & Cytoplasm \\
\hline MaPUB40 & GSMUA_Achr5T21060_001 & chr5:22947743.22958748 reverse & 11,006 & 2412 & 803 & 90.01 & 5.48 & 9 & Nucleus \\
\hline MaPUB41 & GSMUA_Achr5T21360_001 & chr5:23436476..23440174 forward & 3699 & 2280 & 759 & 83.62 & 6.06 & 4 & Cytoplasm, Nucleus \\
\hline MaPUB42 & GSMUA_Achr5T28670_001 & chr5:28750592..28755063 reverse & 4472 & 1713 & 570 & 62.66 & 5.75 & 6 & Nucleus \\
\hline MaPUB43 & GSMUA_Achr6T02470_001 & chr6:1615103..1616422 reverse & 1320 & 1143 & 380 & 40.98 & 8.69 & 3 & Nucleus \\
\hline MaPUB44 & GSMUA_Achr6T06560_001 & chr6:4418997..4420524 forward & 1528 & 1122 & 373 & 40.57 & 7.67 & 2 & Nucleus \\
\hline MaPUB45 & GSMUA_Achr6T10990_001 & chr6:7323129..7324847 reverse & 1719 & 1719 & 572 & 59.6 & 8.22 & 1 & Cytoplasm, Nucleus \\
\hline MaPUB46 & GSMUA_Achr6T11310_001 & chr6:7517739..7519221 reverse & 1483 & 1143 & 380 & 41.25 & 6.73 & 3 & Nucleus \\
\hline MaPUB47 & GSMUA_Achr6T18140_001 & chr6:12201719..12203017 reverse & 1299 & 1230 & 409 & 43.26 & 5.84 & 2 & Nucleus \\
\hline MaPUB48 & GSMUA_Achr6T22980_001 & chr6:22176387..22181121 reverse & 4735 & 1884 & 627 & 69.88 & 8.57 & 10 & Nucleus \\
\hline MaPUB49 & GSMUA_Achr6T25390_001 & chr6:26469894..26479975 reverse & 10,082 & 5637 & 1878 & 202.19 & 5.28 & 7 & Cytoplasm, Nucleus \\
\hline MaPUB50 & GSMUA_Achr6T25670_001 & chr6:26728664..26729950 forward & 1287 & 1287 & 428 & 44.86 & 8.66 & 1 & Nucleus \\
\hline MaPUB51 & GSMUA_Achr6T28500_001 & chr6:28868630..28876365 forward & 7736 & 1833 & 610 & 67.96 & 8.79 & 9 & Nucleus \\
\hline MaPUB52 & GSMUA_Achr6T36530_001 & chr6:34466958.34471270 reverse & 4313 & 1983 & 660 & 72.48 & 6.42 & 6 & Nucleus \\
\hline MaPUB53 & GSMUA_Achr7T04450_001 & chr7:3366660..3370421 forward & 3762 & 2349 & 798 & 88.28 & 7.88 & 11 & Nucleus \\
\hline MaPUB54 & GSMUA_Achr7T05890_001 & chr7:4367855..4369228 forward & 1374 & 1374 & 457 & 48.06 & 9.35 & 1 & Cytoplasm \\
\hline MaPUB55 & GSMUA_Achr7T09440_001 & chr7:7719776..7722069 forward & 2294 & 1917 & 638 & 70.34 & 8.95 & 4 & Nucleus \\
\hline MaPUB56 & GSMUA_Achr7T09650_001 & chr7:7847312..7851814 reverse & 4503 & 2208 & 735 & 82.73 & 8.7 & 10 & $\begin{array}{l}\text { Cell membrane, Chloroplast, } \\
\text { Cytoplasm, Nucleus }\end{array}$ \\
\hline MaPUB57 & GSMUA_Achr7T17740_001 & chr7:20510968..20523478 reverse & 12,511 & 6279 & 2092 & 223.93 & 5.63 & 9 & Cytoplasm, Nucleus \\
\hline MaPUB58 & GSMUA_Achr7T18640_001 & chr7:21413794..21415228 forward & 1435 & 1140 & 379 & 41.34 & 6.41 & 3 & Cell membrane, Nucleus \\
\hline MaPUB59 & GSMUA_Achr7T19940_001 & chr7:22941467..22943503 reverse & 2037 & 1590 & 529 & 55.7 & 8.55 & 3 & Nucleus \\
\hline MaPUB60 & GSMUA_Achr7T22130_001 & chr7:24687902..24690206 reverse & 2305 & 1902 & 633 & 68.33 & 6.17 & 3 & Nucleus \\
\hline MaPUB61 & GSMUA_Achr7T23310_001 & chr7:25579820..25581016 forward & 1197 & 1197 & 398 & 43.4 & 8.95 & 1 & Nucleus \\
\hline MaPUB62 & GSMUA_Achr8T01360_001 & chr8:1146290..1151389 forward & 5100 & 1731 & 576 & 63.53 & 8.57 & 8 & Nucleus \\
\hline MaPUB63 & GSMUA_Achr8T04940_001 & chr8:3264288..3266015 forward & 1728 & 1299 & 432 & 46.78 & 5.51 & 2 & Nucleus \\
\hline MaPUB64 & GSMUA_Achr8T09590_001 & chr8:6746141..6747127 forward & 987 & 987 & 328 & 33.95 & 5.91 & 1 & Nucleus \\
\hline MaPUB65 & GSMUA_Achr8T11510_001 & chr8:8320562..8327700 forward & 7139 & 2552 & 850 & 95.41 & 6.12 & 11 & Nucleus \\
\hline MaPUB66 & GSMUA_Achr8T12630_001 & chr8:9406796..9419285 forward & 12,490 & 1571 & 526 & 57.29 & 6.33 & 18 & Nucleus \\
\hline MaPUB67 & GSMUA_Achr8T25140_001 & chr8:29062381..29066399 reverse & 4019 & 1263 & 420 & 44.9 & 7.71 & 4 & Nucleus \\
\hline MaPUB68 & GSMUA_Achr8T30420_001 & chr8:32504280..32505566 forward & 1287 & 822 & 273 & 29.04 & 8.75 & 3 & Nucleus \\
\hline MaPUB69 & GSMUA_Achr9T00690_001 & chr9:540580..541705 forward & 1126 & 660 & 219 & 23.38 & 9.33 & 2 & Cytoplasm, Nucleus \\
\hline MaPUB70 & GSMUA_Achr9T01670_001 & chr9:1306678..1309170 forward & 2493 & 1401 & 466 & 50.56 & 8.52 & 2 & Nucleus \\
\hline MaPUB71 & GSMUA_Achr9T08750_001 & chr9:5638998..5640625 forward & 1628 & 1038 & 345 & 36.08 & 8.47 & 3 & Nucleus \\
\hline MaPUB72 & GSMUA_Achr9T12570_001 & chr9:8175348..8180293 reverse & 4946 & 903 & 300 & 31.91 & 6.01 & 2 & Nucleus \\
\hline MaPUB73 & GSMUA_Achr9T14450_001 & chr9:9395076..9397076 reverse & 2001 & 1398 & 465 & 49.29 & 8.95 & 5 & Nucleus \\
\hline MaPUB74 & GSMUA_Achr9T14560_001 & chr9:9502271..9504578 reverse & 2308 & 1758 & 585 & 63.11 & 8.67 & 3 & Nucleus \\
\hline MaPUB75 & GSMUA_Achr9T15600_001 & chr9:10315534..10319034 forward & 3501 & 2031 & 676 & 75.01 & 6.85 & 7 & Nucleus \\
\hline
\end{tabular}


Table 1. Cont.

\begin{tabular}{|c|c|c|c|c|c|c|c|c|c|}
\hline $\begin{array}{l}\text { Gene } \\
\text { Name }\end{array}$ & Locus ID & Chromosome Location & $\begin{array}{c}\text { Gene } \\
\text { DNA (bp) }\end{array}$ & $\begin{array}{l}\text { CDS } \\
\text { (bp) }\end{array}$ & $\begin{array}{c}\text { Protein } \\
\text { Length (aa) }\end{array}$ & $\begin{array}{c}\text { Molecular } \\
\text { Weight (kD) }\end{array}$ & $\begin{array}{c}\text { Theoretical } \\
\text { pI }\end{array}$ & Exon & Putative Localization \\
\hline MaPUB76 & GSMUA_Achr9T28820_001 & chr9:32677125..32679340 reverse & 2216 & 1800 & 599 & 64.89 & 9.09 & 4 & Nucleus \\
\hline MaPUB77 & GSMUA_Achr9T29420_001 & chr9:33126026..33130242 reverse & 4217 & 1836 & 611 & 68.18 & 6.64 & 9 & Nucleus \\
\hline MaPUB78 & GSMUA_Achr10T04850_001 & chr10:14480133..14481503 forward & 1371 & 735 & 244 & 26.1 & 4.96 & 2 & Nucleus \\
\hline MaPUB79 & GSMUA_Achr10T09450_001 & chr10:19291616..19292842 forward & 1227 & 1155 & 384 & 40.37 & 8.41 & 2 & Nucleus \\
\hline MaPUB80 & GSMUA_Achr10T11310_001 & chr10:20821803..20823180 forward & 1378 & 1119 & 372 & 39.55 & 6.28 & 3 & Nucleus \\
\hline MaPUB81 & GSMUA_Achr10T17120_001 & chr10:24446918..24449504 reverse & 2587 & 1125 & 374 & 40.87 & 6.14 & 2 & Nucleus \\
\hline MaPUB82 & GSMUA_Achr10T17900_001 & chr10:24916493..24917809 forward & 1317 & 1131 & 376 & 40.82 & 8.25 & 3 & Nucleus \\
\hline MaPUB83 & GSMUA_Achr10T22180_001 & chr10:27439361..27448280 reverse & 8920 & 4041 & 1346 & 148.98 & 5.49 & 14 & Nucleus \\
\hline MaPUB84 & GSMUA_Achr10T22750_001 & chr10:27801994..27810019 forward & 8026 & 2631 & 876 & 98.61 & 6.12 & 11 & Cell membrane, Nucleus \\
\hline MaPUB85 & GSMUA_Achr10T22780_001 & chr10:27821009..27830243 forward & 9235 & 2595 & 864 & 96.02 & 8.5 & 14 & Nucleus \\
\hline MaPUB86 & GSMUA_Achr10T27710_001 & chr10:30760030..30761828 reverse & 1799 & 1359 & 452 & 50.07 & 5.87 & 5 & Nucleus \\
\hline MaPUB87 & GSMUA_Achr11T02530_001 & chr11:1736304..1752382 reverse & 16,079 & 1581 & 526 & 57.22 & 6.41 & 18 & Nucleus \\
\hline MaPUB88 & GSMUA_Achr11T06020_001 & chr11:4516270..4520590 reverse & 4321 & 2040 & 679 & 73.94 & 7.63 & 3 & Nucleus \\
\hline MaPUB89 & GSMUA_Achr11T09460_001 & chr11:7338409..7341969 forward & 3561 & 1278 & 425 & 45.72 & 8.17 & 3 & Nucleus \\
\hline MaPUB90 & GSMUA_Achr11T20980_001 & chr11:21780963..21789676 reverse & 8714 & 2400 & 799 & 89.46 & 5.71 & 9 & Nucleus \\
\hline MaPUB91 & GSMUA_AchrUn_randomT07480_001 & $\begin{array}{l}\text { chrUn_random:32367065..32372845 } \\
\text { forward }\end{array}$ & 5781 & 2403 & 800 & 90.95 & 6.37 & 11 & Nucleus \\
\hline
\end{tabular}




\subsection{Gene Structure and Phylogenetic Analysis of U-Box Gene Family Members}

By comparing the full-length cDNA sequence with the corresponding genomic DNA sequence, the exon-intron structure of each MaU-box was determined. The number of exons in MaU-box genes ranged from 1 to 18 (Figure 2).

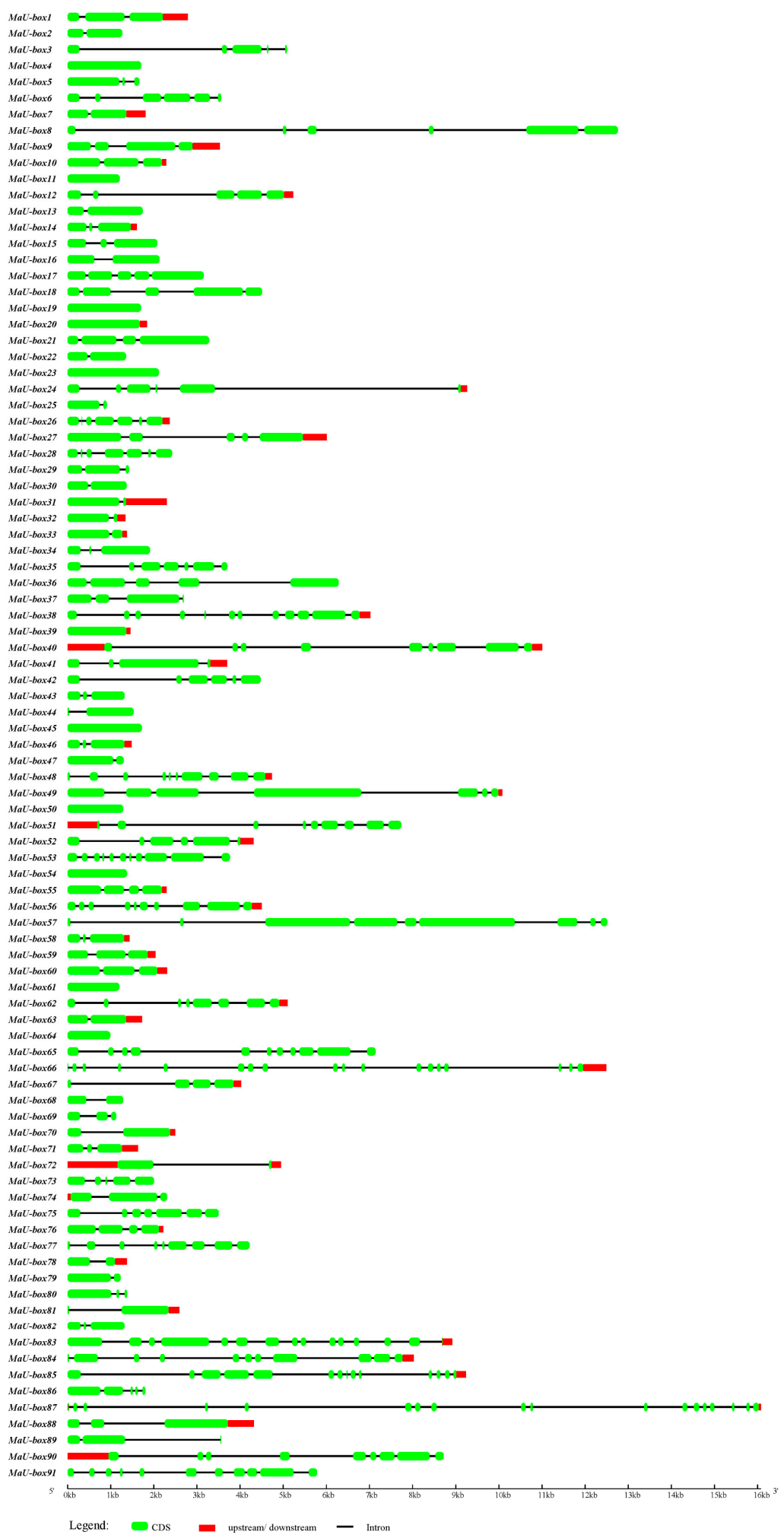

Figure 2. MaU-box gene structures. 
To study the evolutionary relationship of banana U-box proteins, a neighbor-joining (NJ) tree was constructed with U-box proteins from banana, rice, and Arabidopsis (Figure 3). The aa sequences of the U-box of 91 proteins from banana, 61 from Arabidopsis, and 77 from rice were used. Phylogenetic analysis showed that all identified U-box proteins from banana together with Arabidopsis and rice were clearly divided into seven subgroups. Subgroups I, II, III, IV, V, VI, and VII contain 8, 2, 10, 8, 26, 32, and 5 gene family members, respectively. In general, the U-box from banana had a closer relationship with rice compared with Arabidopsis. Interestingly, these MaU-box genes with similar genetic structures are clustered together. For example, MaU-box51/65/84/91 of subgroup I each contain 11 exons, MaU-box66/87 of subgroup II each contain 18 exons, and MaU-box4/19/20/39/45/54 of subgroup III each contain 1 exon.

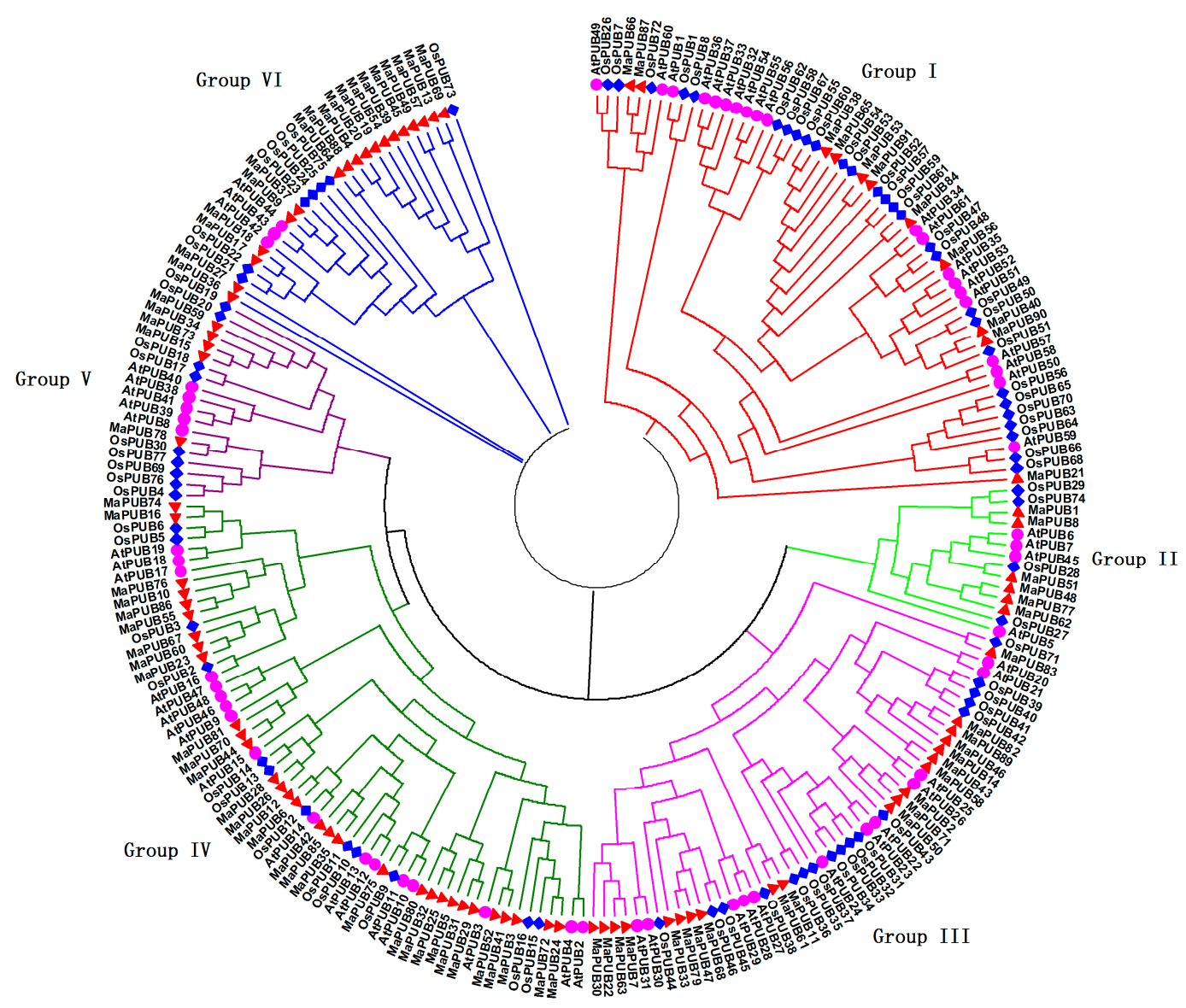

Figure 3. Phylogenetic tree representing relationships among U-box domains of banana, Arabidopsis and rice. The different colors and different numbers (Group I-VI) indicate different MaPUB subgroups. The different shapes indicate different species.

\subsection{Analysis of MaU-Box Gene Family Conserved Motifs}

To investigate the structural diversity and predict the function of MaU-box proteins, 20 conserved motifs in banana U-box were identified using the MEME motif search tool and annotated using SMART tools (Figures 4 and 5). Among the 91 U-box genes, 45 (50\%) contained U-box conservative motifs without ARM motifs, 22 (24\%) contained ARM conservative motifs without U-box motifs, while $24(26 \%)$ contained both U-box conserved motifs and ARM conserved motifs (Figure 6). Motifs 1 and 2 are U-box conservative motifs; motifs 5, 8, 10,13,16, and 18 are ARM conservative motifs; motifs 9 and 14 are Pkinase-Tyr motifs; and motif 6 is an STYKc motif. Motifs 3, 4, 7, 11, 12, 15, 17, 19, and 20 are unknown. The features of 20 motifs are shown in Figure 5. 


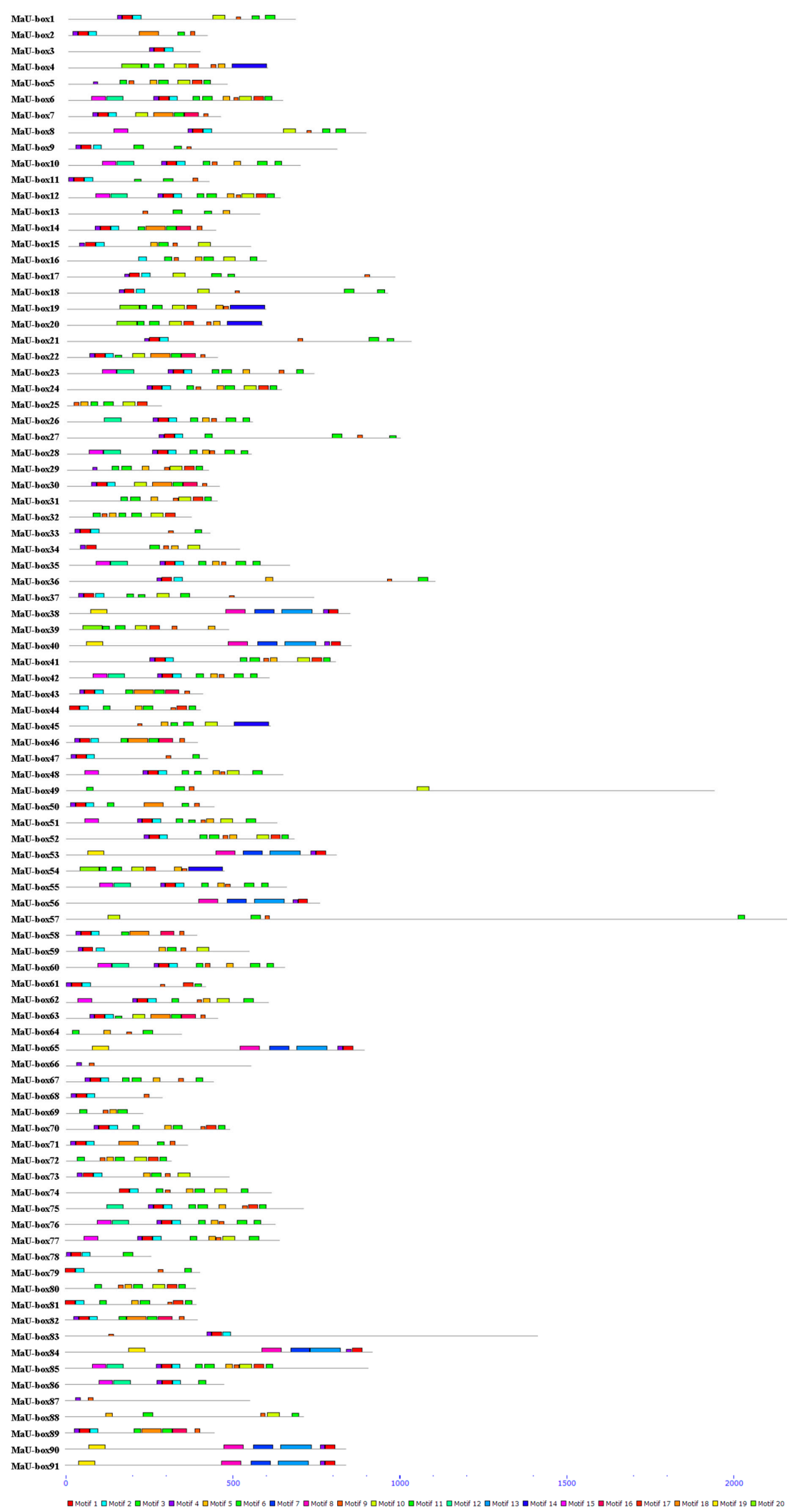

Figure 4. Distribution of conserved motifs for the banana MaU-box proteins. 


\subsection{Expression Profile of MaU-Box Genes in Different Organs}

Figure 7 shows that among the 91 MaU-box genes, 88 were at least expressed in one tissue, occupying $97 \%$ of all gene numbers. Moreover, among the 88 genes, 76 were expressed in all tissues. The Mau-box gene family was differentially expressed in various tissues. This gene family had the highest expression in the roots, where 48 genes exhibited the highest expression. By contrast, the lowest expression was observed in the male flowers, where 17 genes (MaU-box8/9/13/16/17/19/21/ $28 / 30 / 32 / 35 / 37 / 38 / 46 / 50 / 56 / 89)$ exhibited the lowest expression.
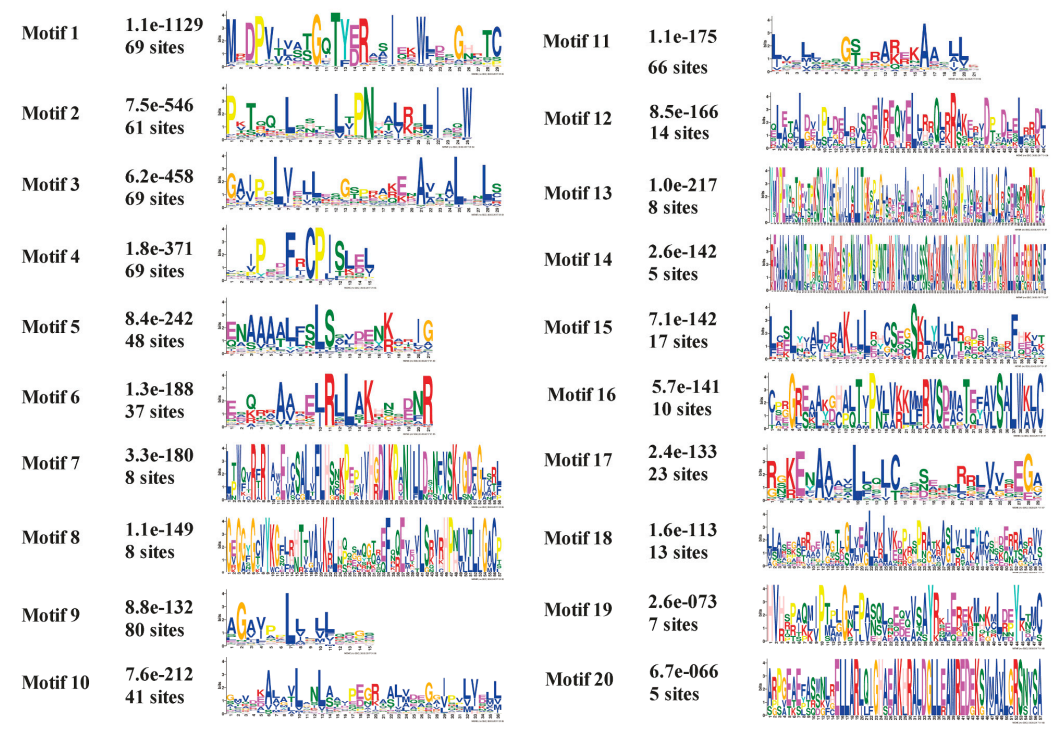

Figure 5. The conserved domains in the MaU-box proteins.

\subsection{Expression Profile of MaU-Box Genes in Fruit Developmental Period}

Figure 8 illustrates that $62 \mathrm{MaU}-$ box genes were at least expressed during one developmental phase, 59 of which were expressed in all developmental phases. The MaU-box gene family was also differentially expressed at various developmental phases of banana. The gene family had the highest expression at the beginning of banana's development phase (day 25), during which 29 genes (MaU-box3/4/8/9/10/13/17/18/19/20/22/25/27/28/34/38/39/41/43/48/49/50/51/52/56/59/61/ $63 / 70$ ) had the highest expression. High MaU-box gene expression was also observed during banana fructescence (day 88), which was second only to the expression on day 25. During the maturity phase of fructescence, 21 genes (MaU-box5/7/12/13/16/21/33/42/47/55/57/65/66/67/69/71/81/84/86/87/88) had the highest expression. Four genes had significant linear expression at the developmental phase. Among them, three genes (MaU-box8/27/61) were gradually downregulated, whereas one gene (MaU-box71) was gradually upregulated with increasing development time. 


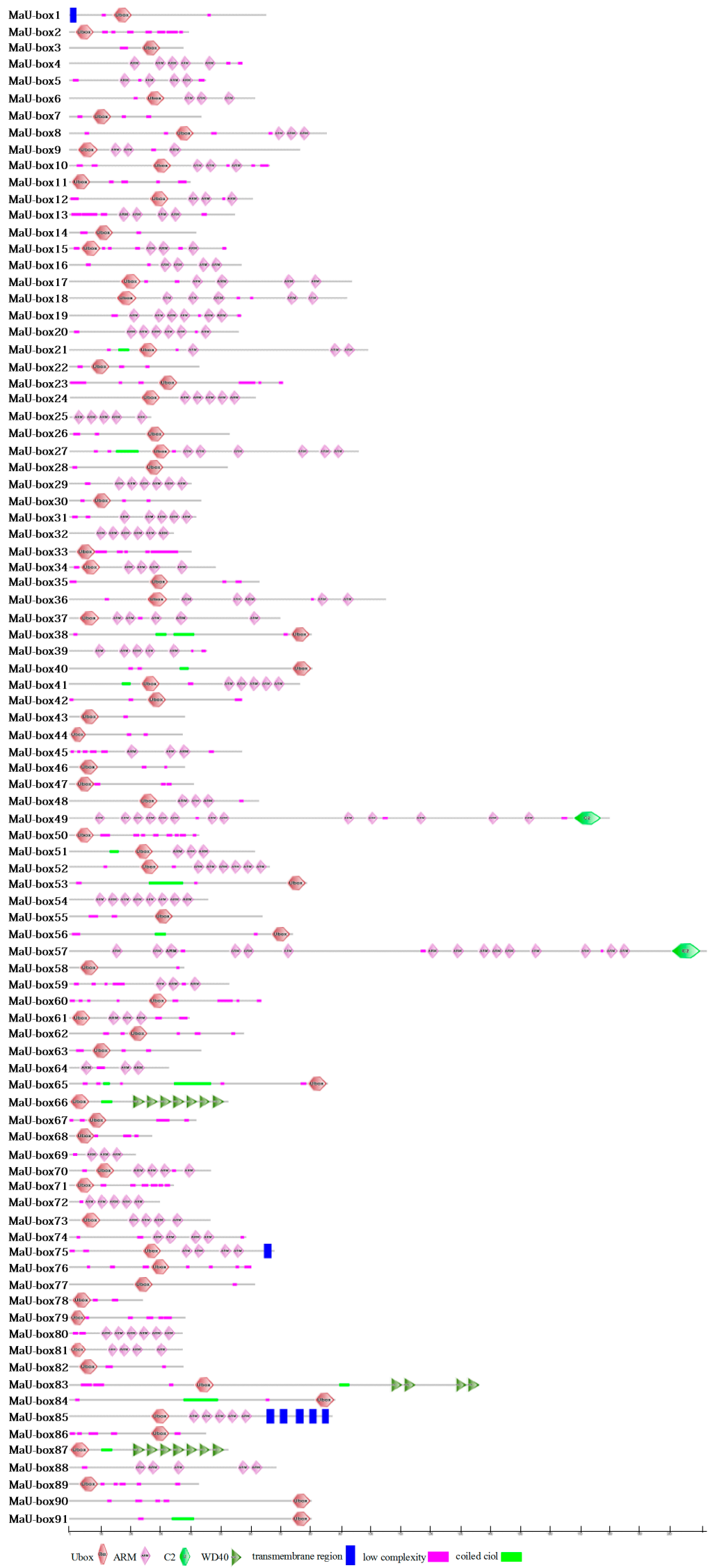

Figure 6. The typical protein structures in the MaU-box proteins. 


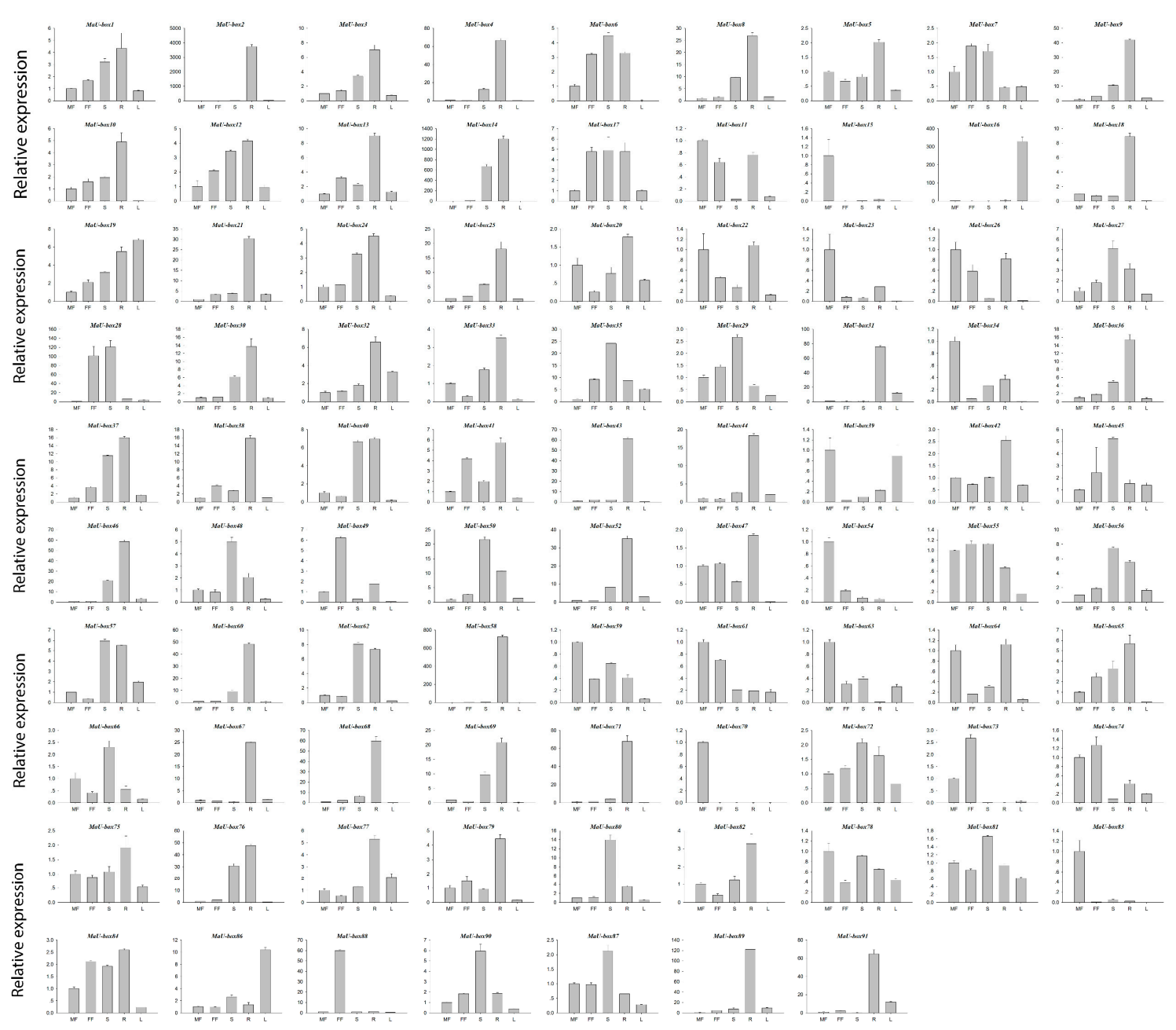

Figure 7. Expression profiles of MaU-box genes in male flowers (MF), female flowers (FF), stems (S), roots $(\mathrm{R})$, and leaves $(\mathrm{L})$. 


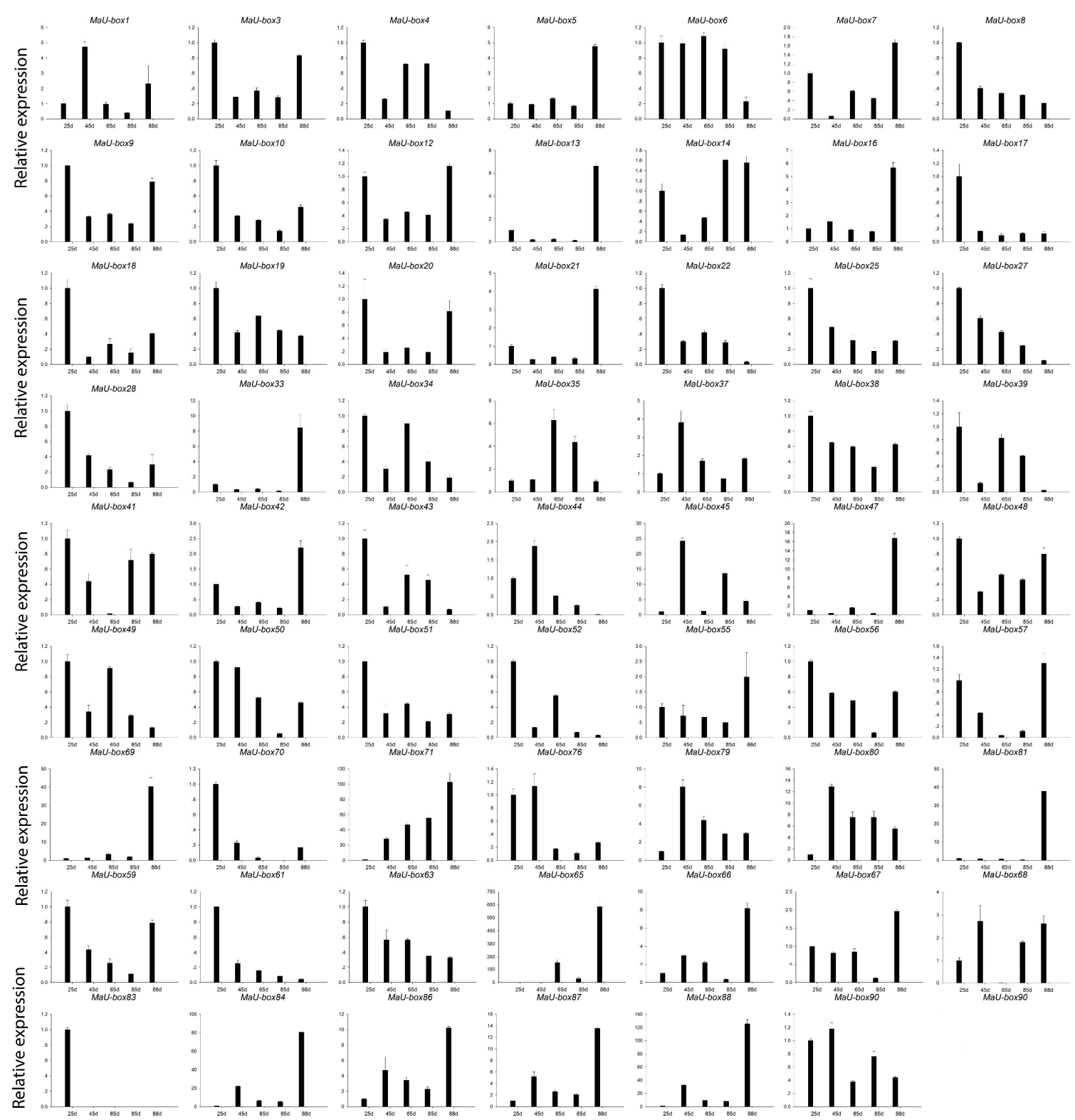

Figure 8. Expression profiles of MaU-box genes in banana pulp during the fruit developmental period. d: day.

\subsection{Differential Expression of MaU-Box Genes under Abiotic Stresses}

Figure 9 shows that $60 \mathrm{MaU}$-box genes responded to drought, salt, and low-temperature stressors. Among these stressors, the MaU-box gene family showed the strongest response to drought. A total of $55 \mathrm{MaU}-b o x$ genes exhibited the highest expression under this stressor, during which 45 genes were upregulated by more than tenfold. The MaU-box gene family showed the highest expression at $24 \mathrm{~h}$, during which 54 genes exhibited the highest expression. Salt stress also resulted in the high regulation of the MaU-box gene family, and this stressor led to the highest expression of four genes (MaU-box63/65/71/78) and the upregulation of two genes (MaU-box63/65) by more than tenfold $(p<0.05)$. 


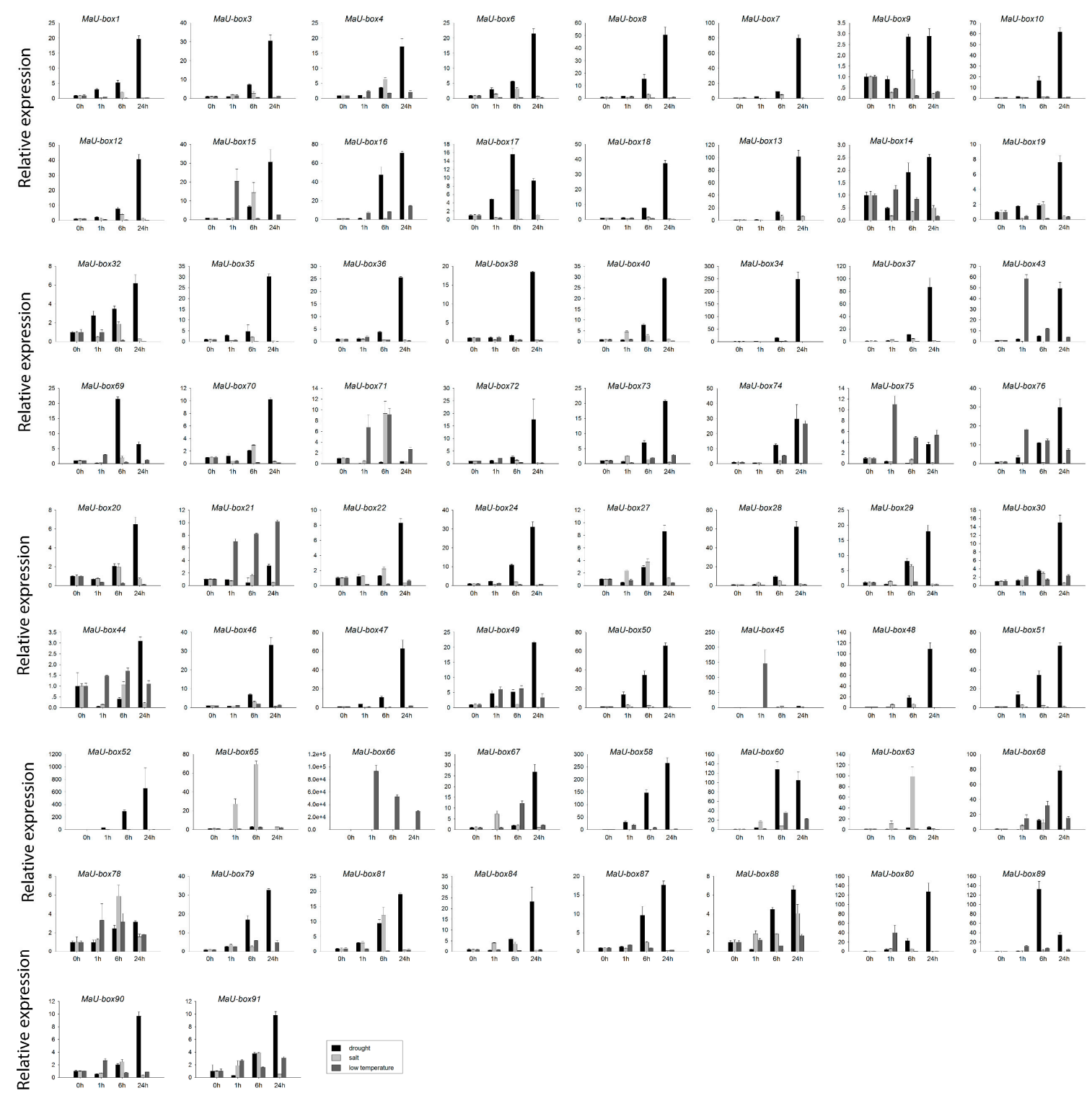

Figure 9. Expression analysis of MaU-box genes in banana leaves under different abiotic treatments.

\section{Discussion}

The characteristics and functions of the U-box gene family has been studied in several plants $[27,28]$. In the present study, systematic phylogenetic analyses were conducted to obtain a detailed classification and nomenclature of the banana U-box. We found 91 PUB (Plant U-box) genes in banana genome. Similarly, 61 U-box proteins of Arabidopsis [12] and 77 U-box-containing proteins of rice had been identified and analyzed [17]. In total, 125 soybean PUB (GmPUB) genes, which encode proteins containing the U-box domain, have been identified [22]. The distribution of U-box proteins among species of different kingdoms is uneven [17]. Our data showed that the banana U-box genes were distributed across all 11 chromosomes at different densities. Phylogenetic analysis of the U-box from banana, Arabidopsis, and rice suggested that the U-box could be clustered into seven subgroups (I-VII). A similar study in soybean found that $125 \mathrm{GmPUB}$ proteins were classified into six groups by phylogenetic analysis [22]. In this study, most banana U-box proteins show closer phylogenetic distance to their putative banana homologs than to their corresponding putative rice and Arabidopsis orthologs. Moreover, the U-box from banana had a closer relationship with rice compared with Arabidopsis. Interestingly, banana proteins MaU-box56, MaU-box78, MaU-box83 and MaU-box84 showed a closer phylogenetic relationship to the rice proteins than to their banana paralogs, suggesting 
that these banana proteins and their corresponding rice orthologs have evolved from a common ancestor before the speciation of the two species [17]. In the present study, conserved motif analysis showed that all identified MaU-box had typical U-box domains. Generally, a protein-protein interaction domain in E3 ubiquitin ligases interacts with their substrates for ubiquitination [29], and a complete U-box domain was found in all PUB proteins [30-32]. The proteins that contained conserved motifs had low sequence similarity, suggesting that mutations were accumulated during evolution [22]. The U-box in banana are found in combination with a variation of domains including armadillo (ARM) repeats, WD40 repeats, the tetratricopeptide (TPR) domain. The ARM repeats have been shown mostly to mediate the interaction with substrates, indicating that interaction renders substrates available for ubiquitination [23]. So the U-box proteins without ARM repeats in banana might have different interactions of E3 ubiquitin ligases with their substrates compared with the U-box proteins containing ARM repeats. The MaU-box gene family was differentially expressed in various tissues of banana. Similarly, several AtPUB-ARM genes were widely expressed in different tissues [33]. The MaU-box gene family had the highest expression in the roots. In a previous study, $12 \mathrm{MaE} 2$ genes had the highest expression levels in roots [24]. These results suggested that MaU-box genes might be involved in the formation of the root system. PUB proteins play important roles in regulating plant growth and development [34]. In the present study, the $29 \mathrm{MaU}$-box genes (MaU-box3/4/8/9/10/13/17/18/19/20/22/25/27/28/34/38/39/41/43/48/49/50/51/52/56/59/61/ $63 / 70$ ) had the highest expression at the beginning of banana's developmental stage (day 25), which could be explained by the fact that the highly expressed genes usually play important roles in plant development [35], suggesting ubiquitination activation through the first stages of fruit development. Of note, the expression of eight MaE2 genes [23] and three MaU-box genes decreased gradually with prolonged developmental time. In strawberry fruit, all the genes decreased gradually after the flowering stage [36]. These data indicated that some genes (e.g., the MaU-box gene) might play important roles for the growth and development of fruits. Studies have shown that the U-box protein is involved in the response to various environmental stresses $[9,20,37]$. The U-box protein gene quickly responded to both biotic stress and abiotic stress and significantly influenced the accumulation of resistance related proteins in grapevine [20]. Silencing tomato U-box E3 ligase ACRE74 lead to break down of Cf9-especified resistance against Cladosporium fulvum leaf mold [20]. The U-box genes of rice might be involved in the defense against diseases [17]. A previous study observed differential expression patterns in nine soybean genes under drought stress [22]. In the present study, the MaU-box genes exhibited stronger response to drought than to salt and low temperature. Under drought stress, $45 \mathrm{MaU}-$ box genes were upregulated by more than tenfold. OsPUB57 showed a stronger expression only in the resistant plants carrying the Pi9-resistant gene [17]. Consistent with this study, in our study, the MaPUB84 and MaPUB91 genes which showed closer phylogenetic distance to OsPUB57 had high expressions under stress. These results indicated that PUB genes might have key functions in responding to drought stress in plant.

\section{Materials and Methods}

\subsection{Plant Materials and Treatment}

The test material "Brazil" banana was obtained from the banana plantation of the National Banana Industry Technical System of Zhanjiang Comprehensive Test Station, South Subtropical Crops Institute, Chinese Academy of Tropical Agricultural Sciences (Zhanjiang, Guangdong, China). Different organs (roots, stems, leaves, female flowers, male flowers) were collected to study the temporal and spatial expression patterns of bananas. The fruits were collected at different developmental stages $(25,45$, 65, and 85 days after florescence) to study fruit development. A healthy and consistent banana seedling with four leaves was selected for stress experiments. The banana seedlings were treated with $20 \%$ PEG 6000 (drought stress treatment) and $200 \mathrm{mM} \mathrm{NaCl}$ (salt stress treatment) and harvested at different time points $(1,6$, and $24 \mathrm{~h})$ after treatment [23]. The experiments were performed in triplicate. 
All samples were frozen in liquid nitrogen and stored at $-80^{\circ} \mathrm{C}$ for the purpose of extracting RNA for expression analysis.

\subsection{Genome Identification of Banana U-Box Gene Family Members}

To identify the potential members of the banana U-box protein family, publish the Arabidopsis thaliana and rice U-box protein sequences as seed sequences, and used BLASTP method search the banana genome database (Banana Genome Hub, available online: http:/ /banana-genome.cirad.fr/ content/download-dh-pahang) and Phytozome (available online: http://www.phytozome.net/) database. All candidate U-box genes were further verified by using SMART conserved domain search tools (available online: http:/ / smart.embl-heidelberg.de/), eliminating repeat sequences, and deleting genes without the U-box domain. The MW and PI prediction of all U-box proteins was performed using the ProtParam tool (available online: http://web.expasy.org/orgparam/). Information on the MaU-box gene, including chromosomal location, DNA sequence, CDS sequence, and aa length, was obtained from phyome12 (available online: https:/ / phytozome.jgi.doe.gov/pz/portal.html\#!info? alias=Org_Gmax). The MW and theoretical PI of the candidate MaU-box protein were obtained using the ExPASy Online Tool (available online: http://expasy.org/tools/). The subcellular localization of banana U-box protein was predicted by using the online software, Plant-mPLoc (available online: http://www.csbio.sjtu.edu.cn/bioinf/plant-multi/\#). Finally, chromosome mapping was performed using the MapInspect tool according to the position of the U-box on the chromosome. For convenience, the MaU-box genes were numbered MaU-box1-MaU-box91 according to the order of chromosome 1 to 11. The structure of each gene was visualized using the Gene Structure Display Server (available online: http://gsds.cbi.pku.edu.cn/).

\subsection{MaU-Box Protein Conserved Motif and Phylogenetic Analysis}

The protein conserved motif of the MaU-box gene family was analyzed using MEME Suite 4.11.4 (available online: http://meme.nbcr.net/meme/) software. The maximum number of protein motifs was 20, and the length of the motifs was 6 to 200 aa.

To understand the evolutionary relationship of the U-box gene, we used the Clustal X version 1.83 software (lllkirch, France) with default parameters to compare the sequences of Arabidopsis thaliana, rice, and banana U-box gene family members. The phylogenetic tree was constructed by comparing the results with MEGA6.0 software (state college, PA, USA). The parameters of the software were set as follows: NJ method as the adjacency method and Poisson correction, paired delete, and bootstrap (1000 repetitions).

\subsection{Gene Expression Analysis}

The MaActin fragment of the banana was selected as the internal reference, and the primers were designed according to the registered sequence. All the MaU-box genes secific primers were designed according to the coding sequences by Primer5 software (PREMIER Biosoft International, Palo Alto, CA, USA) and checked using Blast in NCBI (available online: https:/ / www.ncbi.nlm.nih.gov/). The relative expression level of the U-box gene was calculated using Equation $2^{-\Delta \Delta} \mathrm{Ct}$.

\section{Conclusions}

Ninety-one U-box genes of the banana genome were classified into seven subgroups. Typical U-box domains were found in all identified MaU-box. The MaU-box gene family had the highest expression in the roots, and the strongest expression was found at the first developmental stage. The MaU-box genes exhibited stronger response to drought than to salt and low temperature. The results of this study provide information on the evolution and functions of the MaU-box genes.

Author Contributions: Data curation, H.H.; Formal analysis, D.S., Y.H.; Investigation, C.D.; Project administration, J.X.; Software, C.D.; Writing-original draft, H.H.; Writing-review \& editing, J.X. 
Funding: This research was funded by Modern Agro-industry Technology Research System (Project No.CARS-31-16).

Conflicts of Interest: The authors declare no conflict of interest.

\section{References}

1. Wang, Z.; Tian, X.; Zhao, Q.; Liu, Z.; Li, X.; Ren, Y.; Tang, J.; Fang, J.; Xu, Q.; Bu, Q. The E3 Ligase DROUGHT HYPERSENSITIVE Negatively Regulates Cuticular Wax Biosynthesis by Promoting the Degradation of Transcription Factor ROC4 in Rice. Plant Cell 2017, 228-244. [CrossRef] [PubMed]

2. Peters, J.M. SCF and APC: The Yin and Yang of cell cycle regulated proteolysis. Curr. Opin. Cell Biol. 1998, 10, 759-768. [CrossRef]

3. Vierstra, R.D. The ubiquitin-26S proteasome system at the nexus of plant biology. Nat. Rev. Mol. Cell Biol. 2009, 10, 385-397. [CrossRef] [PubMed]

4. Santner, A.; Estelle, M. The ubiquitin-proteasome system regulates plant hormone signaling. Plant J. 2010, 61, 1029-1040. [CrossRef] [PubMed]

5. Pruneda, J.N.; Littlefield, P.J.; Soss, S.E.; Nordquist, K.A.; Chazin, W.J.; Brzovic, P.S.; Klevit, R.E. Structure of an E3:E2 Ub Complex Reveals an Allosteric Mechanism Shared among RING/U-box Ligases. Mol. Cell 2012, 47, 933-942. [CrossRef] [PubMed]

6. Ciechanover, A. The ubiquitin-proteasome pathway: On protein death and cell life. EMBO J. 1998, 17, 7151-7160. [CrossRef] [PubMed]

7. Zhang, X.; Abrahan, C.; Colquhoun, T.A.; Liu, C.J. A Proteolytic Regulator Controlling Chalcone Synthase Stability and Flavonoid Biosynthesis in Arabidopsis. Plant Cell 2017, 29, 1157-1174. [CrossRef]

8. Finley, D.; Ciechanover, A.; Varshavsky, A. Ubiquitin as a central cellular regulator. Cell 2004, 116, $29-34$. [CrossRef]

9. González-Lamothe, R.; Tsitsigiannis, D.I.; Ludwig, A.A.; Panicot, M.; Shirasu, K.; Jones, J.D.G. The U-Box Protein CMPG1 Is Required for Efficient Activation of Defense Mechanisms Triggered by Multiple Resistance Genes in Tobacco and Tomato. Plant Cell 2006, 18, 1067-1083. [CrossRef]

10. Kelley, D. E3 ubiquitin ligases: Key regulators of hormone signaling in plants. Mol. Cell. Proteom. 2018, 17, 1047-1054. [CrossRef]

11. Moon, J.; Parry, G.; Estelle, M. The Ubiquitin-Proteasome Pathway and Plant Development. Plant Cell 2004, 16, 3181-3195. [CrossRef] [PubMed]

12. Azevedo, C.; Santosrosa, M.J.; Shirasu, K. The U-box protein family in plants. Trends Plant Sci. 2001, 6, 354-358. [CrossRef]

13. Koegl, M.; Hoppe, T.; Schlenker, S.; Ulrich, H.D.; Mayer, T.U.; Jentsch, S. A novel ubiquitination factor, E4, is involved in multiubiquitin chain assembly. Cell 1999, 96, 635-644. [CrossRef]

14. Meacham, G.C.; Patterson, C.; Zhang, W.; Younger, J.M.; Cyr, D.M. The Hsc70 co-chaperone CHIP targets immature CFTR for proteasomal degradation. Nat. Cell Biol. 2001, 3, 100-105. [CrossRef] [PubMed]

15. Stone, S.L.; Anderson, E.M.; Mullen, R.T.; Goring, D.R. ARC1 Is an E3 Ubiquitin Ligase and Promotes the Ubiquitination of Proteins during the Rejection of Self-Incompatible Brassica Pollen. Plant Cell 2003, 15, 885-898. [CrossRef] [PubMed]

16. Cho, S.K.; Kim, W.T. Arabidopsis PUB22 and PUB23 Are Homologous U-Box E3 Ubiquitin Ligases That Play Combinatory Roles in Response to Drought Stress. Plant Cell 2008, 20, 1899-1914. [CrossRef] [PubMed]

17. Zeng, L.R.; Park, C.H.; Venu, R.C.; Gough, J.; Wang, G.L. Classification, Expression Pattern, and E3 Ligase Activity Assay of Rice U-Box-Containing Proteins. Mol. Plant 2008, 1, 800-815. [CrossRef]

18. Hatakeyama, S.; Yada, M.; Matsumoto, M.; Ishida, N.; Nakayama, K.I. U Box Proteins as a New Family of Ubiquitin-Protein Ligases. J. Biol. Chem. 2001, 276, 33111-33120. [CrossRef]

19. Aravind, L.; Koonin, E.V. The U box is a modified RING finger-A common domain in ubiquitination. Curr. Biol. 2000, 10, 132-134. [CrossRef]

20. Jiao, L.; Zhang, Y.; Lu, J. Overexpression of a stress-responsive U-box protein gene VaPUB affects the accumulation of resistance related proteins in Vitis vinifera 'Thompson Seedless'. Plant Physiol. Biochem. 2016, 112, 53-63. [CrossRef]

21. Yang, C.W.; González-Lamothe, R.; Ewan, R.A.; Rowland, O.; Yoshioka, H.; Shenton, M.; Ye, H.; O’Donnell, E.; Jones, J.D.G.; Sadanandom, A. The E3 Ubiquitin Ligase Activity of Arabidopsis PLANT U-BOX17 and Its 
Functional Tobacco Homolog ACRE276 Are Required for Cell Death and Defense. Plant Cell 2006, 18, 1084-1098. [CrossRef] [PubMed]

22. Wang, N.; Liu, Y.; Cong, Y.; Wang, T.; Zhong, X.; Yang, S.; Li, Y.; Gai, J. Genome-Wide Identification of Soybean U-Box E3 Ubiquitin Ligases and Roles of GmPUB8 in Negative Regulation of Drought Stress Response in Arabidopsis. Plant Cell Physiol. 2016, 57, 1189-1209. [CrossRef] [PubMed]

23. Trujillo, M. News from the PUB: Plant U-box type E3 ubiquitin ligases. J. Exp. Bot. 2017, 69, 371-384. [CrossRef] [PubMed]

24. Dong, C.; Hu, H.; Jue, D.; Zhao, Q.; Chen, H.; Xie, J.; Jia, L. The banana E2 gene family: Genomic identification, characterization, expression profiling analysis. Plant Sci. 2016, 245, 11-24. [CrossRef] [PubMed]

25. Fu, X.; Cheng, S.; Liao, Y.; Huang, B.; Du, B.; Zeng, W.; Jiang, Y.; Duan, X.; Yang, Z. Comparative analysis of pigments in red and yellow banana fruit. Food Chem. 2017, 239, 1009-1018. [CrossRef] [PubMed]

26. Dhont, A.; Denoeud, F.; Aury, J.M.; Baurens, F.C.; Carreel, F.; Garsmeur, O.; Noel, B.; Bocs, S.; Droc, G.; Rouard, M.; et al. The banana (Musa acuminata) genome and the evolution of monocotyledonous plants. Nature 2012, 488, 213-217. [CrossRef] [PubMed]

27. Wang, C.; Duan, W.; Riquicho, A.R.; Jing, Z.; Liu, T.; Hou, X.; Li, Y. Genome-wide survey and expression analysis of the PUB family in Chinese cabbage (Brassica rapa ssp. pekinesis). Mol. Genet. Genom. 2015, 290, 2241-2260. [CrossRef] [PubMed]

28. Luo, Q.; Li, Y.; Wang, W.; Fei, X.; Deng, X. Genome-wide survey and expression analysis of Chlamydomonas reinhardtii U-box E3 ubiquitin ligases (CrPUBs) reveal a functional lipid metabolism module. PLoS ONE 2015, 10, e122600. [CrossRef]

29. Patterson, C. A new gun in town: The U box is a ubiquitin ligase domain. Sci. Signal. 2002, 116 , pe4. [CrossRef]

30. Yee, D.; Goring, D.R. The diversity of plant U-box E3 ubiquitin ligases: From upstream activators to downstream target substrates. J. Exp. Bot. 2009, 60, 1109-1121. [CrossRef]

31. Maere, S.; De Bodt, S.; Raes, J.; Casneuf, T.; Van Montagu, M.; Kuiper, M.; Van de Peer, Y. Modeling gene and genome duplications in eukaryotes. Proc. Natl. Acad. Sci. USA 2005, 102, 5454-5459. [CrossRef] [PubMed]

32. Cannon, S.B.; Mitra, A.; Baumgarten, A.; Young, N.D.; May, G. The roles of segmental and tandem gene duplication in the evolution of large gene families in Arabidopsis thaliana. BMC Plant Biol. 2004, 4, 10. [CrossRef] [PubMed]

33. Mudgil, Y.; Goring, D.R. A large complement of the predicted Arabidopsis ARM repeat proteins are members of the U-box E3 ubiquitin ligase family. Plant Physiol. 2004, 134, 59-66. [CrossRef] [PubMed]

34. Luo, J.; Shen, G.; Yan, J.; He, C.; Zhang, H. AtCHIP functions as an E3 ubiquitin ligase of protein phosphatase 2A subunits and alters plant response to abscisic acid treatment. Plant J. 2006, 46, 649-657. [CrossRef] [PubMed]

35. Jue, D.; Sang, X.; Liu, L.; Shu, B.; Wang, Y.; Liu, C.; Xie, J.; Shi, S. Identification of WRKY Gene Family from Dimocarpus longan and Its Expression Analysis during Flower Induction and Abiotic Stress Responses. Int. J. Mol. Sci. 2018, 19, 2169. [CrossRef] [PubMed]

36. Garrido-Bigotes, A.; Figueroa, P.M.; Figueroa, C.R. Jasmonate Metabolism and Its Relationship with Abscisic Acid During Strawberry Fruit Development and Ripening. J. Plant Growth Regul. 2018, 37, 101-113. [CrossRef]

37. Yu, T.C.; Xin, L. Ubiquitination in NB-LRR-mediated immunity. Curr. Opin. Plant Biol. 2012, 15, 392-399.

(C) 2018 by the authors. Licensee MDPI, Basel, Switzerland. This article is an open access article distributed under the terms and conditions of the Creative Commons Attribution (CC BY) license (http://creativecommons.org/licenses/by/4.0/). 